\title{
Unexpectedly high field-effect mobility of a soluble, low molecular weight oligoquaterthiophene fraction with low polydispersity
}

\author{
Patrick Pingel • Achmad Zen • Dieter Neher • \\ Ingo Lieberwirth • Gerhard Wegner • Sybille Allard • \\ Ullrich Scherf
}

Received: 28 July 2008 / Accepted: 24 November 2008 / Published online: 11 December 2008

(C) Springer-Verlag 2008

\begin{abstract}
Layers made from soluble low molecular weight polythiophene PQT-12 with low polydispersity exhibit a highly ordered structure and charge-carrier mobilities of the order of $10^{-3} \mathrm{~cm}^{2} /(\mathrm{V} \mathrm{s})$, which we attribute to its proximity to monodispersity. We propose that polydispersity is a decisive factor with regard to structure formation and transport properties of soluble low molecular weight polythiophenes.
\end{abstract}

PACS 68.55.-a · 73.61.Ph · 78.66.Qn $\cdot$ 81.05.Lg $\cdot$ 85.30.Tv

\section{Introduction}

At present, poly(3-alkylthiophene)s, in particular regioregular poly(3-hexylthiophene) (P3HT), are among the mostly studied materials in the field of solution-based organic electronic devices [1]. Processing from solution allows for the

Electronic supplementary material The online version of this article (http://dx.doi.org/10.1007/s00339-008-4994-0) contains supplementary material, which is available to authorized users.

P. Pingel $\cdot$ D. Neher $(\bowtie)$

Institute of Physics and Astronomy, University of Potsdam, Karl-Liebknecht-Straße 24-25, 14476 Potsdam, Germany

e-mail: neher@uni-potsdam.de

A. Zen

Research and Technology Center, Robert Bosch (South East Asia)

Pte Ltd, 50 Nanyang Drive, Singapore 637553, Singapore

I. Lieberwirth · G. Wegner

Solid State Chemistry, Max Planck Institute for Polymer

Research, Ackermannweg 10, 55128 Mainz, Germany

S. Allard · U. Scherf

Macromolecular Chemistry, University of Wuppertal,

Gauss-Straße 20, 42097 Wuppertal, Germany easy and inexpensive coating of large areas but also introduces a multitude of process parameters. In the case of poly(3-alkylthiophene)s, foregoing investigations have established a close correlation between the fabrication conditions, the resulting layer morphology and the transport properties in organic field-effect transistors (OFETs) [2-9].

Moreover, recent studies have documented a pronounced dependence of the OFET mobility on average chain length. For P3HT and other polythiophenes, the mobility was reported to increase systematically with molecular weight (MW) $[2,6,8,10]$ (see e.g. Fig. 5). Most of these studies used fractions of different MW obtained by solvent extraction. Mobilities of pristine layers spin coated from the lowest MW fraction (with a number-average MW $\left(M_{\mathrm{n}}\right)$ of typically $4000 \mathrm{~g} / \mathrm{mol}$ or lower) were mostly in the range of $10^{-6}-10^{-5} \mathrm{~cm}^{2} /(\mathrm{V} \mathrm{s})$, though higher values have been achieved by Kline et al. [3] under certain preparation conditions (see Table S1 in the Electronic Supplementary Material, ESM). The overall poor performance of short-chain polythiophenes is rather astonishing, since layers made from soluble oligothiophenes with a well-defined length such as $\alpha, \omega$-dihexylsexithiophene exhibit mobilities of more than $10^{-2} \mathrm{~cm}^{2} /(\mathrm{V} \mathrm{s})$ [11]. This discrepancy has not been well addressed in the literature.

Here we present a study of different MW fractions of

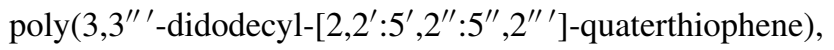
PQT-12. Unlike commonly observed for other polythiophenes, the mobility in these fractions does not decrease monotonically with molecular weight. In fact, the shortchain sample of PQT-12 exhibits an unexpectedly high mobility of $10^{-3} \mathrm{~cm}^{2} /(\mathrm{V} \mathrm{s})$. Optical and structural studies suggest that this anomalous property is related to the very low polydispersity of this fraction. 


\section{Experimental methods}

Poly $\left(3,3^{\prime \prime \prime}\right.$-didodecyl- $\left[2,2^{\prime}: 5^{\prime}, 2^{\prime \prime}: 5^{\prime \prime}, 2^{\prime \prime \prime}\right]$-quaterthiophene), PQT-12, was synthesized in an oxidative polymerization of the corresponding quaterthiophene monomer with $\mathrm{FeCl}_{3}$ according to Ong et al. [12]. The herein investigated PQT12 fractions have been generated by the solvent extraction method as extensively described for regioregular poly(3hexylthiophene), P3HT [6, 13]. The solvents used as well as the molecular weights and the parts by weight of the resulting PQT-12 fractions are listed in Table S2 in the ESM.

The morphologies of thin polymer films on top of $\mathrm{SiO}_{2}$ substrates were characterized by means of tapping-mode atomic force microscopy (AFM) and transmission electron microscopy (TEM). For the TEM measurement the polymer layer was delaminated and transferred onto a carbon-coated copper grid. TEM images were recorded using a LEO 912 TEM setup at an acceleration voltage of $120 \mathrm{kV}$ in the case of P3HT layers and a TECNAI $\mathrm{G}^{2}$ TEM setup at an acceleration voltage of $200 \mathrm{kV}$ in the case of PQT-12 layers.

UV/Vis absorption spectra were recorded using a Perkin Elmer LAMBDA 19 spectrometer. Films were spun onto glass substrates at a constant speed of $1500 \mathrm{rpm}$ from a $10 \mathrm{mg} / \mathrm{ml}$ solution in chloroform. Prior to the process the glass was thoroughly cleaned with several common solvents and silanized in hexamethyldisilazane (HMDS) vapor for $26 \mathrm{~h}$ at a temperature of $60^{\circ} \mathrm{C}$.

Organic field-effect transistor (OFET) properties were prepared in bottom-gate, top-source/drain geometry. Homogeneous polymer layers were formed by spin coating at $1500 \mathrm{rpm}$ from a $10 \mathrm{mg} / \mathrm{ml}$ chloroform solution onto $\mathrm{n}$ doped silicon substrates which are covered with an approx. 300-nm-thick insulating $\mathrm{SiO}_{2}$ layer. Prior to the processing, the $\mathrm{SiO}_{2}$ surface was thoroughly cleaned with several common solvents and has undergone an oxygen plasma treatment ( $5 \mathrm{~min}$ at a power of $200 \mathrm{~W}$ ) followed by silanization using HMDS for $26 \mathrm{~h}$ at $60^{\circ} \mathrm{C}$. Finally, 100 -nm-thick evaporated interdigitating gold electrodes served as source and drain contacts. Device characteristics were recorded using an Agilent 4155C semiconductor parameter analyzer. Preparation and measurements were performed in inert nitrogen atmosphere. Charge-carrier mobilities were calculated from the saturation regions of the output characteristics (drain current vs. source-drain voltage) according to

$I_{\mathrm{DS}, \mathrm{sat}}=\frac{W C_{\mathrm{i}}}{2 L} \mu_{\mathrm{sat}}\left(V_{\mathrm{G}}-V_{\mathrm{T}}\right)^{2}$,

where $W=14.85 \mathrm{~cm}$ and $L=100 \mu \mathrm{m}$ are the channel width and length, respectively, $C_{\mathrm{i}}=11.9 \mathrm{nF} / \mathrm{cm}^{2}$ is the capacitance per unit area and $V_{\mathrm{T}}$ is the threshold voltage.

\section{Results and discussion}

The chemical structures of PQT-12 and P3HT are drawn in Fig. 1 and Table 1 lists the relevant properties of the investigated fractions. In general, the molecular weights obtained by subsequent fractionation are within the range reported for other polythiophenes. However, a peculiarity of our low-MW PQT-12 fraction is its very low polydispersity, implying a very discrete chain-length distribution. A HPLC/MALDI-TOF-MS (matrix-assisted laser desorption/ionization-time-of-flight mass spectroscopy coupled with high-pressure liquid chromatography) analysis showed that roughly $85 \%$ of the low-MW PQT-12 sample consists of the dimers of the quaterthiophene building block. Apart from that, there are oligomers containing 12 or 16 thiophene units present (quaterthiophene trimers and tetramers). The very narrow MW distribution especially for the low-MW PQT-12 fraction is a result of the synthetic scheme: the use of preformed quaterthiophene monomers in the generation of PQT-12 automatically rules out the formation of oligothiophene species with a number of thiophene rings other than ' $4 n$ '. A subsequent solvent extraction with well-suited solvents allows for a complete removal of monomeric quaterthiophene traces and for the isolation of a low-MW PQT-12 fraction which contains mainly the dimeric octathiophene.

The surface morphologies of PQT-12 thin films on top of silicon dioxide substrates were characterized by means

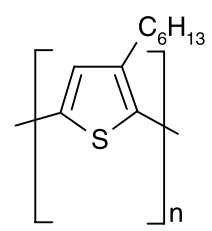

1

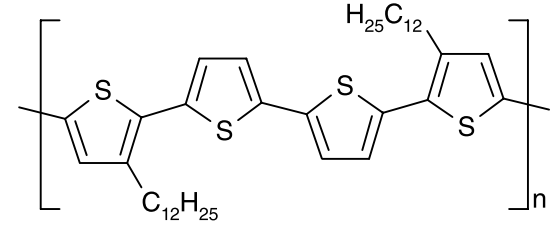

2
Fig. 1 Chemical structures of P3HT (1) and PQT-12 (2)

Table 1 Overview of the investigated compounds of PQT-12 and the opposed P3HT fractions with respect to weight-average $\left(M_{\mathrm{W}}\right)$ and number-average $\left(M_{\mathrm{n}}\right)$ molecular weights and polydispersity index (PDI)

\begin{tabular}{lrrrl}
\hline Compound & \multicolumn{1}{l}{$\begin{array}{l}M_{\mathrm{w}} \\
(\mathrm{g} / \mathrm{mol})\end{array}$} & $\begin{array}{l}M_{\mathrm{n}} \\
(\mathrm{g} / \mathrm{mol})\end{array}$ & PDI & $\begin{array}{l}\text { Notation } \\
(\text { see text })\end{array}$ \\
\hline PQT-12 & 1700 & 1650 & 1.03 & Low MW \\
PQT-12 & 7000 & 6000 & 1.17 & Medium MW \\
PQT-12 & 9000 & 7000 & 1.29 & \\
PQT-12 & 12000 & 10000 & 1.20 & \\
PQT-12 & 26500 & 16000 & 1.66 & High MW \\
P3HT & 3150 & 2200 & 1.43 & Low MW \\
P3HT & 6600 & 5600 & 1.18 & Medium MW \\
P3HT & 25650 & 19000 & 1.35 & High MW \\
\hline
\end{tabular}


Fig. 2 AFM topology (left-hand column) and phase (right-hand column) images of low (a), medium (b) and high (c) molecular weight PQT-12 films on silicon dioxide and corresponding root-mean-square roughnesses (RMS). The lateral dimensions are $3 \times 3 \mu^{2}$
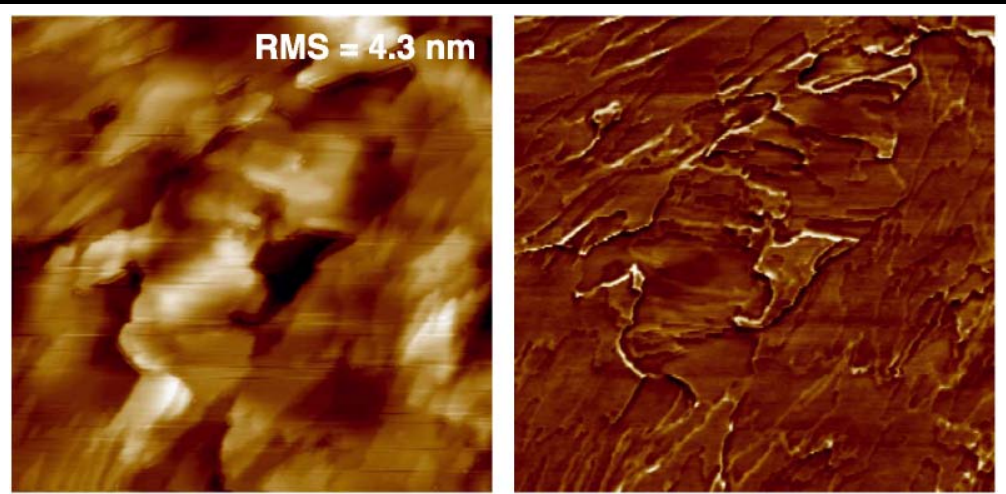

a
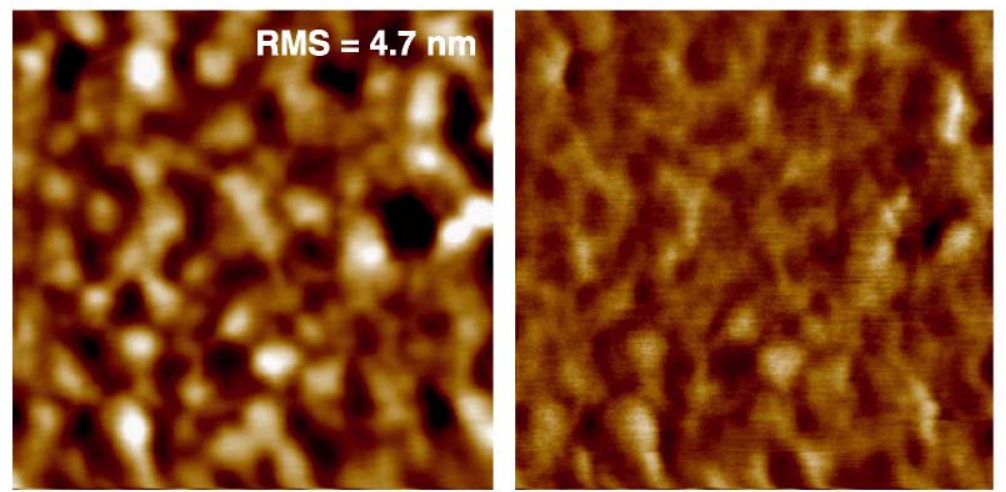

b
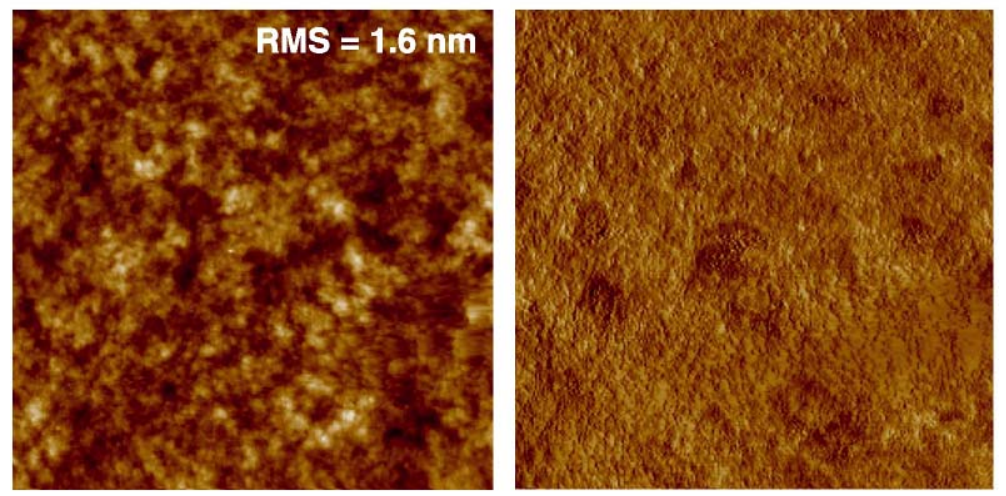

c

of tapping-mode AFM. Figure 2 shows the topography and phase images of low-, medium- and high-MW PQT-12 films. The sharp contours of micrometer-sized crystallites at the surface of low-MW PQT-12 films suggest that these layers predominantly consist of well-ordered crystallites with a large extent in every direction. In contrast, the isotropic nodule-like surface morphologies of medium- and high-MW PQT-12 layers indicate the absence of any long-range order.

Figure 3 (left-hand column) shows the transmission electron microscopy (TEM) images of the low- and high-MW PQT-12 films. In low-MW PQT-12, clearly distinguished crystallites of several micrometers in size adjoin closely or overlap. Using the smallest selective area diffraction aperture $(10 \mu \mathrm{m})$, it was possible to obtain characteristic single crystalline diffraction patterns from only one crystalline do- main (left-hand inset of Fig. 3a). Note that the slight distortion of the point-shaped reflections might be an indication for a slight misalignment within a single crystal causing a textured pattern. The exact analysis of the diffraction pattern in terms of molecular packing and orientation is beyond the scope of this article, but the considerations as described in the ESM indicate that the backbones are oriented parallel to the substrate.

In contrast to this, layers of high-MW PQT-12 embed small crystalline domains with a size of the order of only $10 \mathrm{~nm}$ in a volume of amorphous material. A nanoscopically structured multiphase morphology can be deduced from the comparison of the bright- and dark-field images - the former is caused by electrons transmitted through the amorphous phase without scattering, the latter emerges by the scattering 
Fig. 3 TEM bright-field images of PQT-12 (left-hand column) and $\mathrm{P} 3 \mathrm{HT}$ (right-hand column) thin films of the low (a) and high (b) MW fractions. The insets towards the center of the figure show the electron-diffraction patterns of the corresponding films. The insets at the bottom of (b) show the dark-field images of the high-MW films for easy identification of the embedded crystalline domains in the same scale as the bright-field images

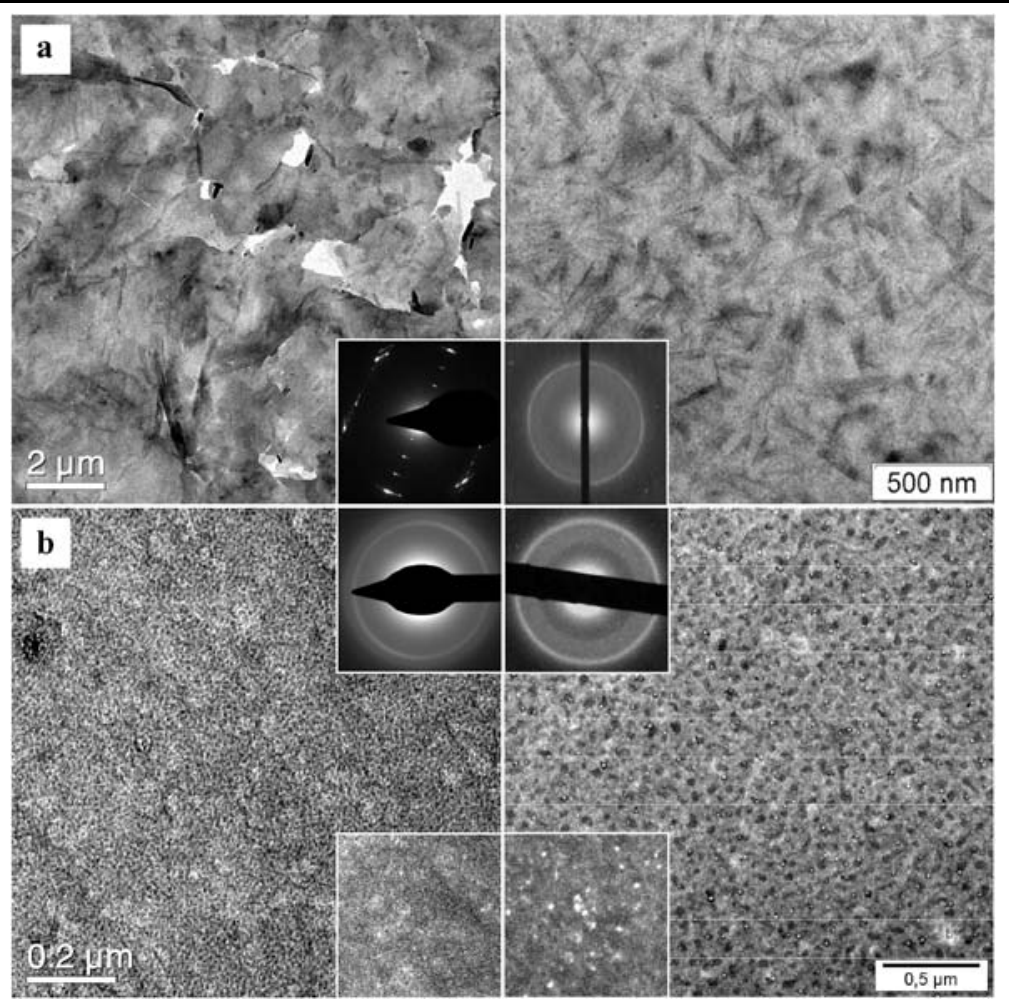

of electrons at crystalline domains. Note that the crystalline orientation in the bulk is random, giving rise to a circular electron-diffraction pattern (left-hand inset of Fig. 3b). We also note that the morphology of our low-MW PQT-12 thin film is distinctly different from the structure of the corresponding low- and high-MW P3HT layers (right-hand image in Fig. 3a).

The very high structural order of the layers formed from low-MW PQT-12 was further confirmed by optical spectroscopy. Figure 4 a shows the absorption spectra of PQT12 thin films with three selected molecular weights. The absorption spectra of medium- and high-MW PQT-12 thin films resemble well those of the corresponding P3HT samples (see Fig. 4b) with regard to the spectral position and the resolution of the vibrational transitions. Compared to its P3HT counterpart, the absorption of the high-MW PQT-12 film is slightly narrower, implying a somewhat higher intrachain order. For both PQT-12 and P3HT the spectra become blue shifted and rather featureless when going from high to medium MW. For P3HT these changes have been explained by a smaller crystallinity of the medium-MW layers, implying that a larger number of chains are contained in disordered areas and that these chains exhibit a less planarized backbone conformation [6, 7]. While decreasing the MW of P3HT further to $2200 \mathrm{~g} / \mathrm{mol}$ renders the absorption even less featureless and blue shifted, the trend is reversed for short-chain PQT-12: low-MW PQT-12 exhibits a narrow and well-structured absorption spectrum, with its maximum even slightly red shifted compared to the medium-
MW fraction. The pronounced resolution of the vibronic transition can be interpreted in terms of the prevalence of a rigid planarized chain conformation and high solid-state order $[9,14,15]$.

At this point the question arises of whether the ability of low-MW PQT-12 to form micrometer-sized singlecrystalline domains (in contrast to P3HT) is due to its specific chemical structure or results from the very low dispersity of this fraction. Kline et al. have observed micrometersized molecular terraces in layers formed from high-MW poly-(2,5,-bis(3-alkylthiophene-2-yl)thieno[3,2-b]thiophene) (pBTTT-C 12 ) [16]. This particular property of pBTTT-C 12 was attributed to substantial interdigitation of the dodecyl side chains, which enforces a three-dimensional ordering of the polymer chains. Though X-ray scattering and infraredabsorption studies suggested a similar degree of local ordering in PQT-12, large-scale ordering has never been reported in PQT-12 layers, to the best of our knowledge.

We would also like to point out that as-prepared PQT-12 with moderate and high MW behaves quite similar to the corresponding P3HT with respect to the optical and structural properties. This leads to the conclusion that the particular ability of low-MW PQT-12 to form micron-scale single crystals is related to its very low polydispersity and not to its specific chemical structure.

It is a well-accepted view that the morphology of lowMW P3HT layers is semicrystalline, characterized by crystalline nanofibrils with rather uniform width, separated by disordered zones $[3,7,8,17]$. Here, the polymer backbones 


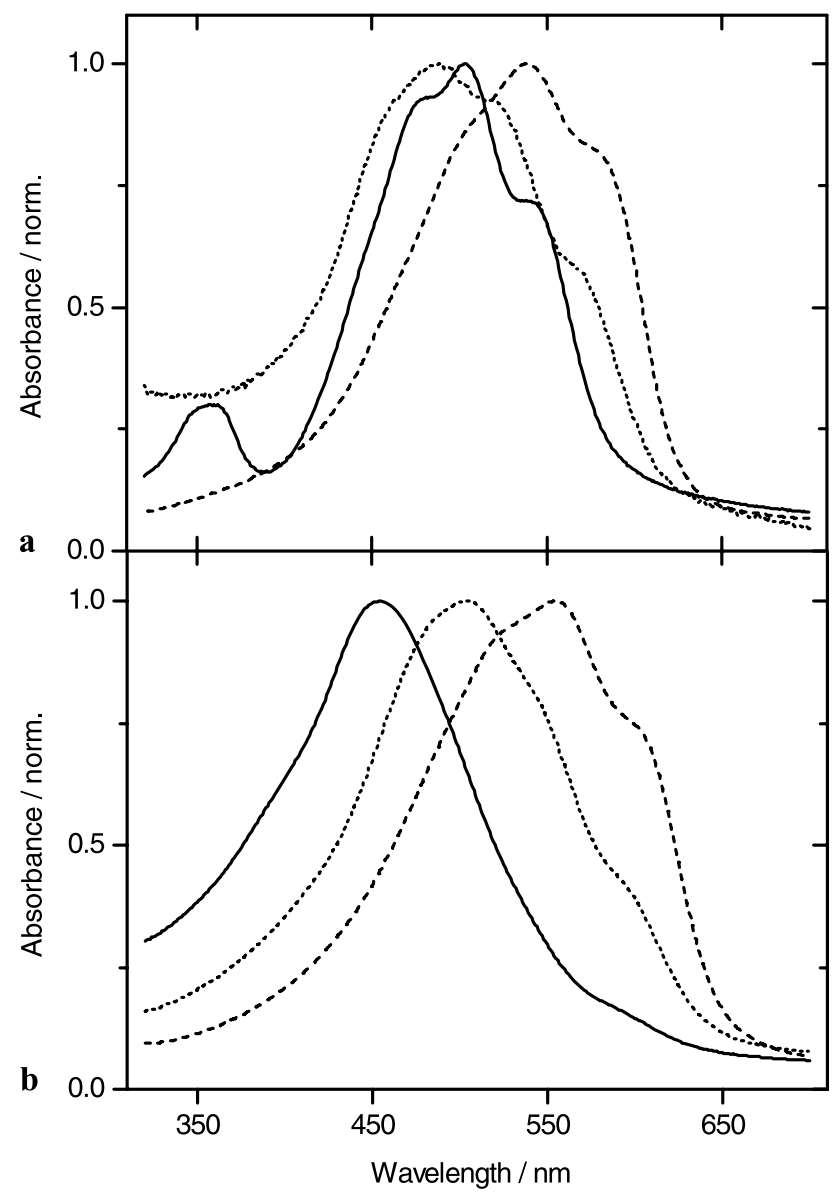

Fig. 4 Absorption spectra of PQT-12 (a) and P3HT (b) thin films. Solid, dotted and dashed lines refer to low, medium and high molecular weight fractions, respectively (see Table 1). The data for P3HT are taken from our former investigation (see [6])

are oriented perpendicular to the nanofibril axis while the amorphous areas host the chain ends. Such a morphology with lamellae of well-ordered chains separated by amorphous interlamellar regions was also observed for epitaxially grown samples of higher MW P3HT [8, 17] and, more generally, in layers of other rigid-rod polymers $[18,19]$. It is due to the fact that chain ends interrupt the highly periodic and evenly spaced sequence of monomer units along the backbone while the variation in chain length, due to polydispersity, inhibits the formation of single-crystalline structures which places chain ends in a regularly spaced fashion. As a consequence, these chain ends will be expelled from the crystalline domains. It is believed that the so-formed amorphous interlamellar regions are the main reason for the low mobility of low- or moderate-MW polythiophenes $[7,17,20]$.

In order to address this issue, we have fabricated OFETs from different MW fractions of PQT-12 and P3HT. All devices were prepared under identical conditions, without further annealing of the polymer layer. Mobilities determined

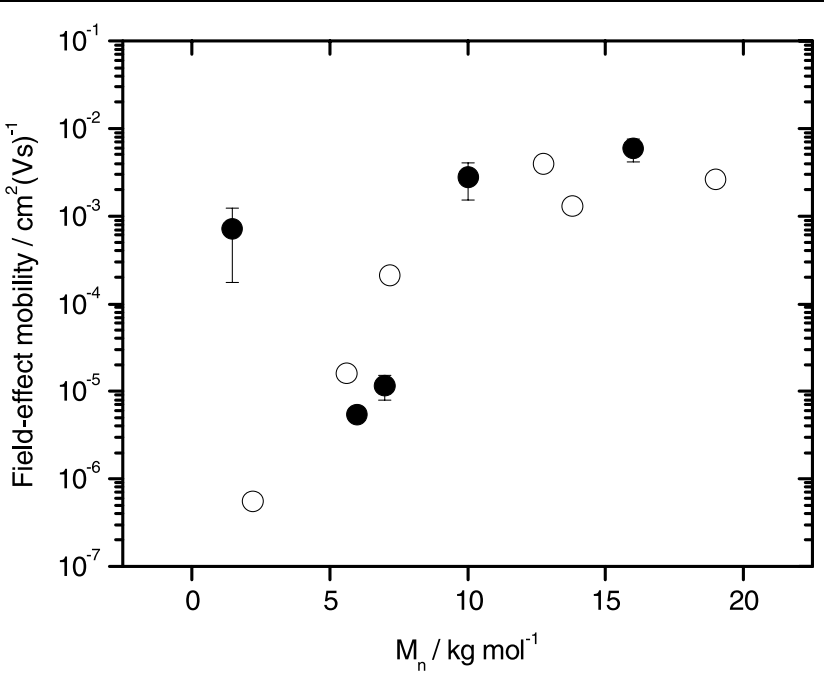

Fig. 5 Field-effect mobility in thin films of PQT-12 (solid symbols) and P3HT (open symbols) against number-average molecular weight. The data for P3HT are taken from our former investigation (see [6])

from the output characteristics of these transistors are plotted as a function of molecular weight in Fig. 5. Note that the mobilities of low- and high-MW PQT-12 are averages over measurements on 10 transistors while the values for medium MW are averages obtained on six different samples.

For high and medium MW, the average mobilities of PQT-12 and P3HT layers are quite comparable. This is expected since our structural and optical studies did not reveal any significant differences regarding layer morphology and chain conformation. For both materials, the mobility of the high-MW samples is of the order of $10^{-2} \mathrm{~cm}^{2} /(\mathrm{V} \mathrm{s})$, but drops by three orders of magnitude when the average chain length is reduced by a factor of nearly four. The mobility of the high-MW PQT-12 fraction is in accordance with Ong et al., who reported the formation of highly ordered layers from a compound with $M_{\mathrm{n}}=17300 \mathrm{~g} / \mathrm{mol}$ and a high field-effect mobility of $0.02-0.05 \mathrm{~cm}^{2} /(\mathrm{V} \mathrm{s})$ [12]. Most importantly, the mobility of our PQT-12 with the lowest MW $\left(M_{\mathrm{n}}=1650 \mathrm{~g} / \mathrm{mol}\right)$ is close to $10^{-3} \mathrm{~cm}^{2} /(\mathrm{V} \mathrm{s})$, more than three orders of magnitude higher than that of the corresponding low-MW P3HT $\left(M_{\mathrm{n}}=2200 \mathrm{~g} / \mathrm{mol}\right)$, and it almost reaches the values measured for high-MW materials.

This finding highlights the importance of disordered intergrain boundaries in determining the mobility of polythiophene layers. Due to the narrow chain-length distribution of our low-MW PQT-12 sample, the driving force for forming those amorphous interlamellar regions between the crystalline domains is largely reduced and carriers can be transported efficiently over distances. To substantiate the impact of polydispersity further, it would be desirable to study polyand oligothiophenes with a fixed MW and selected polydispersity systematically. However, currently it is not possible to control polydispersity during polymerization as far as needed for such an experiment. 
In conclusion, we have analyzed the structural, optical and electrical properties of fractions of PQT-12 with different molecular weight. Surprisingly, low-MW PQT-12 exhibits a highly ordered morphology with micrometersized single-crystalline domains. Charge-carrier mobilities measured in bottom-gate OFET devices were close to $10^{-3} \mathrm{~cm}^{2} /(\mathrm{V} \mathrm{s})$, more than three orders of magnitude higher than that of the corresponding low-MW P3HT. We attribute these particular properties to the very low polydispersity of this fraction, which is a consequence of the synthetic route used. Presumably, polydispersity is one of the decisive factors with regard to structure formation in thin solid layers, which needs to be carefully considered in the interpretation of mobility data from soluble oligo- and polythiophenes.

Acknowledgement We thank the German Science Foundation for funding within SPP 1121.

\section{References}

1. A. Facchetti, Mater. Today 10, 28 (2007)

2. R.J. Kline, M.D. McGehee, E.N. Kadnikova, J.S. Liu, J.M.J. Frechet, Adv. Mater. 15, 1519 (2003)

3. R.J. Kline, M.D. McGehee, E.N. Kadnikova, J.S. Liu, J.M.J. Frechet, M.F. Toney, Macromolecules 38, 3312 (2005)

4. H. Sirringhaus, Adv. Mater. 17, 2411 (2005)

5. H.C. Yang, T.J. Shin, L. Yang, K. Cho, C.Y. Ryu, Z.N. Bao, Adv. Funct. Mater. 15, 671 (2005)

6. A. Zen, J. Pflaum, S. Hirschmann, W. Zhuang, F. Jaiser, U. Asawapirom, J.P. Rabe, U. Scherf, D. Neher, Adv. Funct. Mater. 14, 757 (2004)
7. A. Zen, M. Saphiannikova, D. Neher, J. Grenzer, S. Grigorian, U. Pietsch, U. Asawapirom, S. Janietz, U. Scherf, I. Lieberwirth, G. Wegner, Macromolecules 39, 2162 (2006)

8. R. Zhang, B. Li, M.C. Iovu, M. Jeffries-El, G. Sauve, J. Cooper, S.J. Jia, S. Tristram-Nagle, D.M. Smilgies, D.N. Lambeth, R.D. McCullough, T. Kowalewski, J. Am. Chem. Soc. 128, 3480 (2006)

9. J.F. Chang, J. Clark, N. Zhao, H. Sirringhaus, D.W. Breiby, J.W. Andreasen, M.M. Nielsen, M. Giles, M. Heeney, I. McCulloch, Phys. Rev. B 74, 115318 (2006)

10. J.M. Verilhac, R. Pokrop, G. LeBlevennec, I. Kulszewicz-Bajer, K. Buga, M. Zagorska, S. Sadki, A. Pron, J. Phys. Chem. B 110, 13305 (2006)

11. H.E. Katz, J.G. Laquindanum, A.J. Lovinger, Chem. Mater. 10, 633 (1998)

12. B.S. Ong, Y.L. Wu, P. Liu, S. Gardner, J. Am. Chem. Soc. 126, 3378 (2004)

13. M. Trznadel, A. Pron, M. Zagorska, Macromolecules 31, 5051 (1998)

14. J. Gierschner, J. Cornil, H.-J. Egelhaaf, Adv. Mater. 19, 173 (2007)

15. G. Li, V. Shrotriya, J. Huang, Y. Yao, T. Moriarty, K. Emery, Y. Yang, Nat. Mater. 4, 864 (2005)

16. R. Kline, D. DeLongchamp, D. Fischer, E. Lin, L. Richter, M. Chabinyc, M. Toney, M. Heeney, I. McCulloch, Macromolecules 40, 7960 (2007)

17. M. Brinkmann, P. Rannou, Adv. Funct. Mater. 17, 101 (2007)

18. G. Lieser, M. Oda, T. Miteva, A. Meisel, H.-G. Nothofer, U. Scherf, D. Neher, Macromolecules 33, 4490 (2000)

19. W. Wang, G. Lieser, G. Wegner, Macromolecules 27, 1027 (1994)

20. S. Joshi, S. Grigorian, U. Pietsch, P. Pingel, A. Zen, D. Neher, U. Scherf, Macromolecules 41, 6800 (2008) 